\title{
Recovering TMS-evoked EEG responses masked by muscle artifacts
}

\section{Mutanen, Tuomas $\mathrm{P}$.}

2016-10-01

Mutanen, T P , Kukkonen, M , Nieminen , J O , Stenroos , M , Sarvas , J \& Ilmoniemi , R J 2016 , ' Recovering TMS-evoked EEG responses masked by muscle artifacts ' , Neurolmage , vol. 139 , pp. 157-166 . https://doi.org/10.1016/j.neuroimage.2016.05.028

http://hdl.handle.net/10138/225039

https://doi.org/10.1016/j.neuroimage.2016.05.028

publishedVersion

Downloaded from Helda, University of Helsinki institutional repository.

This is an electronic reprint of the original article.

This reprint may differ from the original in pagination and typographic detail.

Please cite the original version. 


\title{
Recovering TMS-evoked EEG responses masked by muscle artifacts
}

\author{
Tuomas P. Mutanen ${ }^{\mathrm{a}, \mathrm{b}, *}$, Matleena Kukkonen ${ }^{\mathrm{a}, \mathrm{b}}$, Jaakko O. Nieminen ${ }^{\mathrm{a}, \mathrm{b}}$, Matti Stenroos ${ }^{\mathrm{a}}$, \\ Jukka Sarvas ${ }^{\text {a }}$, Risto J. Ilmoniemi ${ }^{\text {a,b }}$ \\ a Department of Neuroscience and Biomedical Engineering, Aalto University School of Science, P.O. Box 12200, FI-00076 AALTO, Finland \\ b BioMag Laboratory, HUS Medical Imaging Center, University of Helsinki and Helsinki University Hospital, P.O. Box 340, FI-00029 HUS, Finland
}

\section{A R T I C L E I N F O}

Article history:

Received 16 February 2016

Revised 21 April 2016

Accepted 9 May 2016

Available online 9 June 2016

\section{Keywords:}

Transcranial magnetic stimulation

Electroencephalography

Muscle artifact

Signal-space projection

Minimum-norm estimate

\begin{abstract}
A B S T R A C T
Combined transcranial magnetic stimulation (TMS) and electroencephalography (EEG) often suffers from large muscle artifacts. Muscle artifacts can be removed using signal-space projection (SSP), but this can make the visual interpretation of the remaining EEG data difficult. We suggest to use an additional step after SSP that we call source-informed reconstruction (SIR). SSP-SIR improves substantially the signal quality of artifactual TMS-EEG data, causing minimal distortion in the neuronal signal components.

In the SSP-SIR approach, we first project out the muscle artifact using SSP. Utilizing an anatomical model and the remaining signal, we estimate an equivalent source distribution in the brain. Finally, we map the obtained source estimate onto the original signal space, again using anatomical information. This approach restores the neuronal signals in the sensor space and interpolates EEG traces onto the completely rejected channels.

The introduced algorithm efficiently suppresses TMS-related muscle artifacts in EEG while retaining well the neuronal EEG topographies and signals. With the presented method, we can remove muscle artifacts from TMS-EEG data and recover the underlying brain responses without compromising the readability of the signals of interest.
\end{abstract}

(c) 2016 Elsevier Inc. All rights reserved.

\section{Introduction}

Transcranial magnetic stimulation (TMS) is a non-invasive method to artificially activate the cortex by applying brief and strong magnetic pulses to the brain (Barker et al., 1985). Navigation enables precise targeting of the stimulation to desired cortical areas (e.g., Massimini et al., 2005; Julkunen et al., 2009). By combining navigated TMS with simultaneous electroencephalography (EEG) (Virtanen et al., 1999), we can measure directly how the TMS-evoked activity spreads in the brain. This makes TMS-EEG a useful method for studying effective connectivity (Ilmoniemi et al., 1997; Komssi et al., 2002; Massimini et al., 2005).

So far, TMS-EEG has mainly been used to study relatively medial cortical areas that do not lie directly under cranial muscles (Nikulin et al., 2003; Rosanova et al., 2009; Cona et al., 2011; Farzan et al., 2013). In principle, TMS-EEG could be used to study excitability and connectivity anywhere in the cortex, but this is often challenging because of the TMS-evoked muscle artifacts that are likely to occur when lateral areas are stimulated (Mutanen et al., 2013; Rogasch et al.,

\footnotetext{
* Corresponding author at: Aalto University School of Science, Department of Neuroscience and Biomedical Engineering, Espoo, P.O. Box 12200, FI-00076 AALTO, Finland.

E-mail address: tuomas.mutanen@aalto.fi (T.P. Mutanen).
}

2013). Muscle artifacts often have 10 to 1000 times larger amplitudes than neuronal components and can last tens of milliseconds after the pulse (Mutanen et al., 2013). With some subjects, even the stimulation of more medial areas, such as primary motor cortex (M1), may result in severe muscle-artifact contamination (Mutanen et al., 2013). Therefore, effective artifact-removal methods are needed to improve the usability of TMS-EEG.

Independent component analysis (ICA) has been suggested to separate TMS-EEG data to brain and muscle-artifact signal components (Korhonen et al., 2011; Hernandez-Pavon et al., 2012; Rogasch et al., 2014). However, when using ICA, we have to make a strong assumption that the TMS-evoked muscle and brain responses are statistically independent. Another possible technique is the signal-space-projection (SSP) approach (Mäki and Ilmoniemi, 2011; Hernandez-Pavon et al., 2012; ter Braack et al., 2013). In SSP, we estimate the signal subspace containing the muscle artifacts and form a linear operator that removes most of the artifact from the measured signal. Mäki and Ilmoniemi (2011) showed that SSP is capable of suppressing muscle-artifact components in TMS-EEG signals. However, SSP tends to attenuate also other signals in the sensors close to the origin of the artifact. This makes the conventional interpretation of EEG waveforms and topographies difficult after applying SSP.

In this paper, we solve the SSP-related attenuation problem by using the suppressed data (and the suppressed lead fields) to compute source 
estimates that can be used to reconstruct artifact-free versions of the original neuronal EEG field patterns. We call this method sourceinformed reconstruction (SIR).

With simulations and analysis of measured EEG data, we show that the combined SSP-SIR is able to considerably suppress muscle artifacts while preserving the underlying neural responses of interest. We argue that the present algorithm provides a highly useful tool in analyzing artifactual TMS-EEG data.

\section{Methods}

In this section, we introduce the theoretical basis for the artifact suppression and source-informed data reconstruction. We also describe how we measured, simulated, and analyzed data to validate the approach.

\section{Theory}

We assume a linear data model for the measured EEG:

$\mathbf{S}=\mathbf{L X}+\mathbf{A}+\mathbf{N}$

where $\mathbf{S}$ is the signal matrix whose entry $S_{i, t}$ contains the measured value of channel $i$ at time $t, \mathbf{X}$ is the source matrix whose entry $X_{j, t}$ describes the activity level of a source $j$ at a time $t$, and $\mathbf{L}$ is the lead-field matrix whose entry $L_{i, j}$ determines the sensitivity of channel $i$ to source $j$. The elements $A_{i, t}$ and $N_{i, t}$ of matrices $\mathbf{A}$ and $\mathbf{N}$ describe muscle artifact and noise contamination in $S_{i, t}$, respectively.

We can consider our EEG data lying in a multidimensional signal space where the dimension equals the number of EEG channels. When using SSP, we estimate two signal subspaces where the first is able to explain most of the artifact variance, the second being its orthogonal complement (Uusitalo and Ilmoniemi, 1997). We refer to the former as artifact subspace and the latter as brain subspace, although the artifact subspace is likely to contain a considerable amount of brain signal and vice versa. Once we have estimated the subspaces, we can construct a linear operator $\mathbf{P}$ that projects the data onto the brain subspace, discarding all information in the artifact subspace. Assuming an ideal operator, $\mathbf{P A}=0$, we obtain from Eq. (1)

$$
\mathbf{P S}=\mathbf{P L X}+\mathbf{P A}+\mathbf{P N}=\mathbf{P L X}+\mathbf{P N} .
$$

Eq. (2) has the same form as an equation describing an artifact-free EEG measurement, where PS and PL are the new measurement and lead-field matrices, respectively. By using the $l^{2}$-minimum-norm estimate (MNE) (Hämäläinen and Ilmoniemi, 1994), we obtain an estimate $\hat{\mathbf{X}}$ for the source distribution:

$\hat{\mathbf{X}}=(\mathbf{P L})^{\dagger} \mathbf{P S}$,

where $(\mathbf{P L})^{\dagger}$ is the appropriately regularized pseudoinverse of $\mathbf{P L}$.

Next, we correct the SSP-caused distortions in the signals of interest with SIR; we calculate the signal matrix $\tilde{\mathbf{S}}$ in the original signal space generated by source estimate $\hat{\mathbf{X}}$ :

$\tilde{\mathbf{S}}=\mathbf{L} \hat{\mathbf{X}}$

A similar approach has been previously used in magnetocardiography (Numminen et al., 1995; Burghoff et al., 2000) and in magnetoencephalography (Uutela et al., 2001). Aside from being free from artifacts (exactly true if $\mathbf{P A}=0$ ), $\tilde{\boldsymbol{S}}$ also contains less noise than $\mathbf{S}$. By regularizing Eq. (3) we do not map $\mathbf{S}$ onto the least significant lead-field directions that mainly explain noise $\mathbf{N}$. This reduces the effect of noise on the source estimate $\hat{\mathbf{X}}$, and thus, also on $\tilde{\mathbf{S}}$.
All in all, we can write the whole artifact (and noise) removal process with one equation:

$\tilde{\mathbf{S}}=\mathbf{L}(\mathbf{P L})^{\dagger} \mathbf{P S}$

where $\tilde{\mathbf{S}}$ is the reconstructed, artifact-free data. We refer to the procedure described by Eq. (5) as SSP-SIR.

An essential question is of course how to find $\mathbf{P}$. In this paper, we use the approach suggested by Mäki and Ilmoniemi (2011), which is based on the difference in the frequency spectra between muscle artifacts and neuronal EEG signals. Fig. 1 shows a time-frequency plot of an EEG channel measuring both TMS-evoked neuronal activity and a muscle artifact. During the time when the artifact is present, the signal covers a much broader band than at other times, implying that the highfrequency parts are mostly due to the muscle activation and noise. This is also supported by the findings of Rosanova et al. (2009), who studied the frequency content of TMS-evoked EEG in various cortical locations. Furthermore, the general understanding is that EEG mainly reflects synchronous post-synaptic currents in neuronal populations (Nunez and Silberstein, 2000; Baillet et al., 2001) and that this activity is seen in EEG mainly below $100 \mathrm{~Hz}$ (Buzsáki and Draguhn, 2004). Thus, by taking the data corresponding to the time interval when the muscle artifact is present and filtering it with an appropriate highpass filter $H$ that satisfies $H(\mathbf{L X}) \approx 0$, we obtain from Eq. (1)

$H(\mathbf{S}) \approx H(\mathbf{A})+H(\mathbf{N})$.

We assume that the low-frequency parts of the muscle artifact lie in the same signal subspace with the high-frequency parts. Then, we can estimate the artifact subspace by using the high-passed data. We first write the high-passed signals in terms of the singular value decomposition (SVD):

$H(\mathbf{S})=\mathbf{U} \mathbf{\Sigma} \mathbf{V}^{\mathrm{T}}$,

where $\Sigma$ has the singular values in a descending order on its diagonal and $\mathbf{U}$ and $\mathbf{V}$ have the left and right singular vectors as columns, respectively. The column vectors of $\mathbf{U}$ form an orthonormal basis for the signal space. Because the first singular directions (the leftmost column vectors of $\mathbf{U}$ ) explain most of the variance of the high-passed data and the power of the artifact is expected to dominate the noise during the first few tens of milliseconds, we obtain a good approximation for the

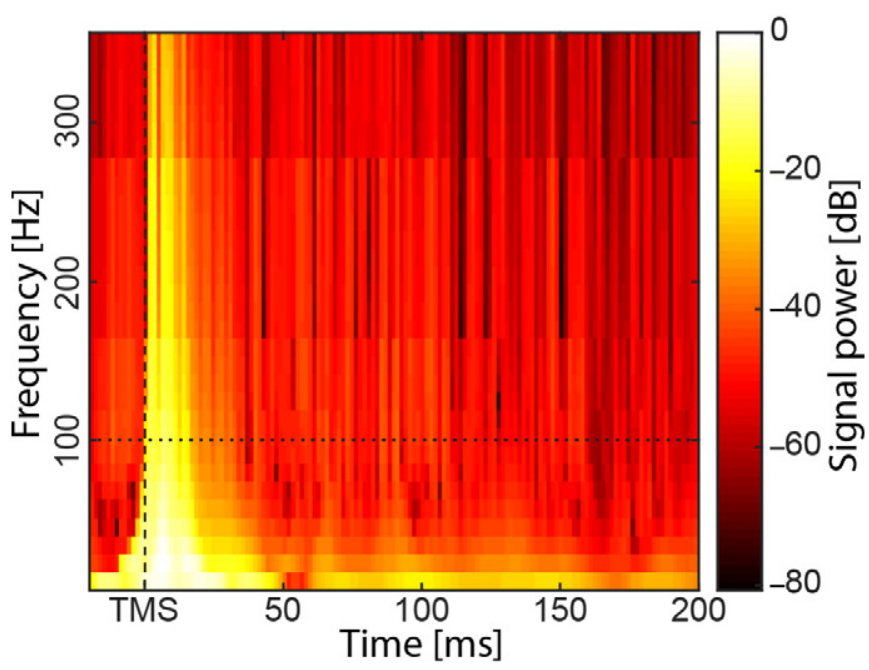

Fig. 1. A time-frequency plot of a typical TMS-evoked EEG response in an artifactcontaminated channel (subject 2, channel C1). We estimate the artifact subspace by high-pass filtering the data from $100 \mathrm{~Hz}$. The plot was made using EEGLAB toolbox (Delorme and Makeig, 2004) with the Morlet-wavelet decomposition. 
high-passed artifact using the truncated SVD:

$$
H(\mathbf{A}) \approx \mathbf{U}_{k} \Sigma_{k} \mathbf{V}_{k}^{\mathrm{T}},
$$

where the matrices $\mathbf{U}_{k}, \mathbf{\Sigma}_{k}, \mathbf{V}_{k}$ consist of the $k$ leftmost columns of $\mathbf{U}, \mathbf{\Sigma}$, and $\mathbf{V}$, respectively. This means that we can approximate the artifact subspace by spanning it with the first $k$ column vectors of $\mathbf{U}$ and that we can suppress the artifacts using the projection matrix $\mathbf{P}$ :

$$
\mathbf{P}=\mathbf{I}-\mathbf{U}_{k} \mathbf{U}_{k}{ }^{\mathrm{T}} .
$$

We now summarize the cleaning of TMS-EEG data with the help of the SSP-SIR method:

1. Remove bad channels ${ }^{1}$ from the analysis.

2. Compute the projection matrix $\mathbf{P}$ using the high-passed data.

3. Multiply the data and lead-field matrices with $\mathbf{P}$ to suppress the effect of muscle artifacts in the source estimation.

4. Compute the source estimates using the suppressed data, PS, and the suppressed lead-field matrix, PL.

5. Use the original lead-field matrix and the obtained source estimates to reconstruct the evoked brain activity in the original signal space.

The cleaning procedure is also illustrated in Fig. 2.

\section{TMS-EEG measurements}

To test the presented algorithm in a realistic situation, we delivered TMS to M1, a cortical area that is commonly studied with TMS-EEG. Although parts of M1 are located close to the vertex of the head, its stimulation may result in a severe muscle-artifact contamination (Mutanen et al., 2013).

We measured three healthy right-handed male subjects: S1, S2, and S3 (ages: 23, 25, and 26 years), who gave their written consent before the experiment. The study was accepted by the Ethics Committee of the Hospital District of Helsinki and Uusimaa and it followed the Declaration of Helsinki. The subjects sat on a comfortable seat and were asked to fixate their eyes at a cross on the wall. Biphasic TMS pulses were delivered using the navigated Nexstim eXimia system (Nexstim Plc, Helsinki, Finland) with a figure-of-eight coil (outer loop diameter $70 \mathrm{~mm}$ ). Simultaneously, TMS-compatible Nexstim eXimia EEG and electromyography (EMG) systems, which use sample-andhold circuitry to block the TMS-induced artifact (Virtanen et al., 1999), were used. The EEG system had 60 electrodes on the scalp in a 10-20 montage. The reference electrode was attached on the right mastoid and the ground was set on the skin over the right cheek bone. Additionally, two electrooculography channels were set on the left side of the left eye and above the right eye to detect possible blinks and eye movements. All electrodes were prepared so that the impedance was below $15 \mathrm{k} \Omega$, preferably below $5 \mathrm{k} \Omega$. The EEG signals were band-pass-filtered at $0.1-350 \mathrm{~Hz}$ and digitized at $1450 \mathrm{~Hz}$. The EMG electrodes were attached to the right abductor pollicis brevis muscle (APB) in a bellytendon montage. To minimize the auditory response in the EEG resulting from the TMS coil click (Nikouline et al., 1999; ter Braack et al., 2015), we delivered white noise to the subjects' ears. The subjects were asked to adjust the volume as high as they comfortably could (however, we controlled that the sound-pressure level never exceeded $90 \mathrm{~dB}$ by calibrating the player with a phantom ear). For navigation, we used T1-weighted magnetic resonance images (MRI), taken with 1-mm MPRAGE sequence (Siemens Espree 1.5-T device was used to scan S2 and Siemens Skyra 3-T device to scan S1 and S3).

\footnotetext{
${ }^{1}$ With bad channels, we mean channels that, based on their neighboring channels, do not reliably measure the potential on the head with respect to the reference. Hence, a signal can be severely contaminated by muscle artifacts without being labeled as bad.
}

The experiment started by determining the resting motor threshold ${ }^{2}$ (MT) intensity (Rossini et al., 1994), which was found to be $47-65 \mathrm{~V} / \mathrm{m}$ and $45-47 \%$ of the maximum stimulator output, depending on the subject. Subsequently, the subjects received 100 TMS pulses (random interstimulus interval: 2-3 s) targeted to the APB hot spot with $100 \%$ MT intensity. If the stimulation of APB hot spot failed to evoke clear muscle artifacts, we moved the coil further away from the vertex of the head along the central sulcus until cranial muscles produced maximum voltages of $\sim 100 \mu \mathrm{V}$ in the EEG signal. If the coil position was changed, the stimulation intensity was adjusted with the help of the navigation so that the induced electric field at the new stimulation site equaled the induced field on the APB hot spot at the MT intensity. When delivering TMS, the navigation-estimated direction of the maximal TMS-induced electric field was always adjusted to be perpendicular with respect to the central sulcus.

\section{Computation of lead fields}

For simulations and SIR, we formed subject-specific, realistic leadfield matrices as follows. We used FreeSurfer (Fischl, 2012) to preprocess and segment the T1-weighted MRI volumes of the subjects to form detailed surfaces of the grey-white-matter boundaries. The inner and outer surfaces of the skull and the scalp surface were built using a combination of BrainSuite (Shattuck and Leahy, 2002) and MNE software (Gramfort et al., 2014) to produce individual surfaces that best fitted the anatomy of the subject close to the EEG sensor array. The EEG array was co-registered with the anatomical model by using the MNE software and the fiducial information imported from the Nexstim neuronavigation system. The skull and scalp surfaces were decimated to 5120 triangles and imported to MATLAB 2015b (The Mathworks Inc., Natick, MA, USA) environment where the surfaces were smoothed and corrected for possible morphological flaws using iso2mesh MATLAB library (Fang and Boas, 2009). Finally, the lead fields were computed using the boundary-element method formulated with the isolated-source, linear Galerkin approach (Stenroos and Sarvas, 2012). We discretized the primary-current distribution as a set of current dipoles oriented perpendicularly with respect to the grey-white-matter surface. 5124 current dipoles were located in the vertices of the greywhite-matter-surface mesh. The conductivities of the brain, skull, and skin were set to be $0.33 \mathrm{~S} / \mathrm{m}, 0.0033 \mathrm{~S} / \mathrm{m}$, and $0.33 \mathrm{~S} / \mathrm{m}$, respectively. The conductivities were chosen based on previous literature where the soft-tissue-skull resistivity ratio has been estimated to be 20-80 (Cohen and Cuffin, 1983; Gonçalves et al., 2003; Lai et al., 2005) and the recent recommendations by Stenroos and Nummenmaa (2016)) who showed that the omission of cerebrospinal fluid in three-layer models can be partially compensated by decreasing the skull conductivity by a factor of 2 .

We also built a spherical three-layer head model that would be common to all subjects, to test how a simplified forward model that still captures the essential physical aspects of the head would perform in SIR. The model consisted of three concentric layers, the brain, the skull, and the scalp (Rush and Driscoll, 1969), which had outer radii of 81, 85 , and $88 \mathrm{~mm}$, respectively. The corresponding conductivities were the same as above. To compute the lead-field matrix, we projected Nexstim's EEG-channel configuration on the spherical scalp and placed 5000 evenly distributed radial current dipoles inside the brain, $76 \mathrm{~mm}$ away from the origin. This is a sufficient equivalent model for a spherical case meaning that all the observed EEG patterns can be explained with an appropriate combination of radial dipoles. The equations needed to compute the spherical-model lead fields can be found in Appendix A.

Finally, we constructed three additional head models to evaluate the importance of including the essential physical aspects of the measurement geometry. The simplest model consisted of the electrodes and

\footnotetext{
2 TMS intensity required to produce 5 motor-evoked potentials in the right APB with peak-to-peak amplitude $\geq 50 \mu \mathrm{V}$ out of 10 stimulations.
} 

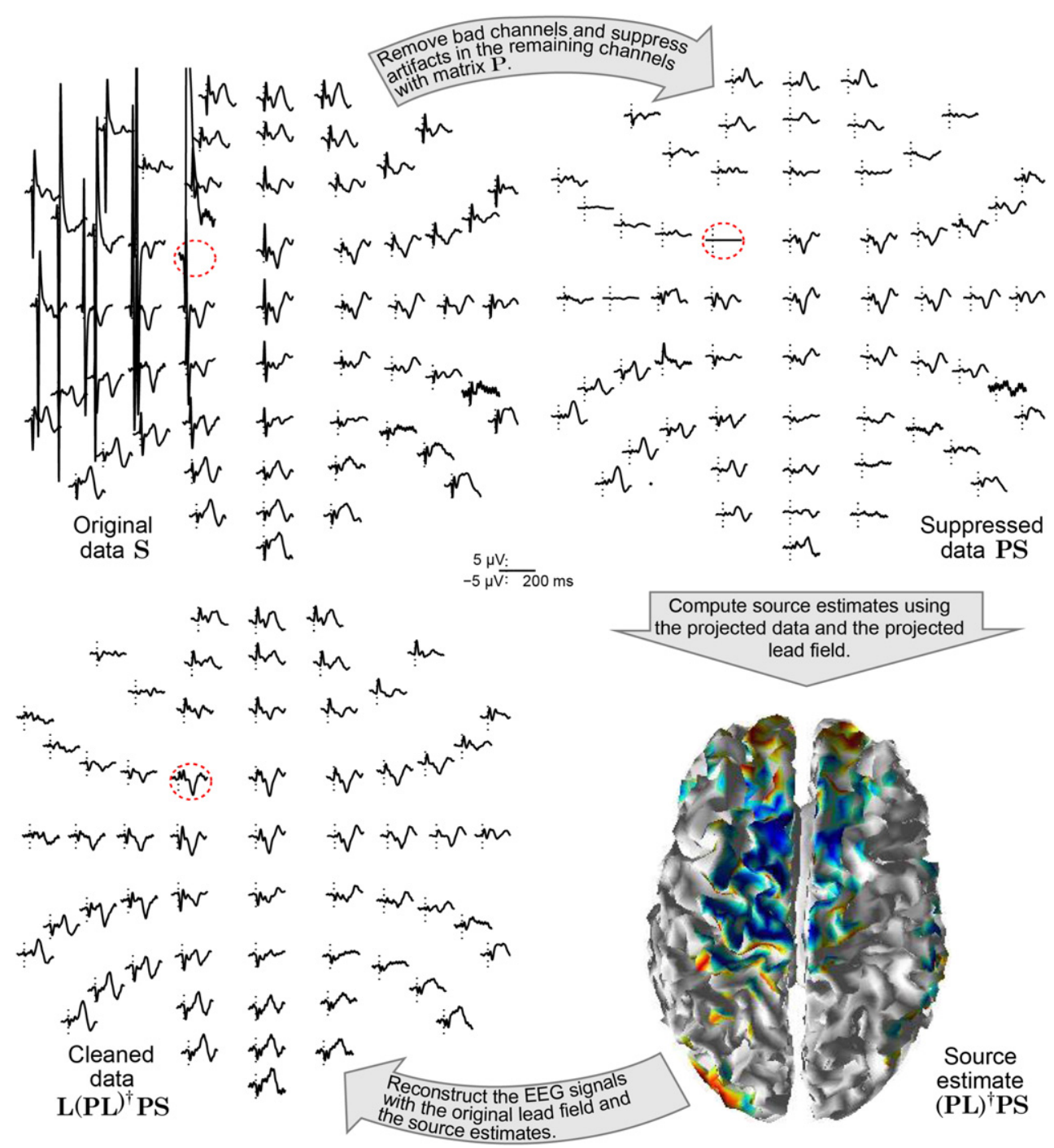

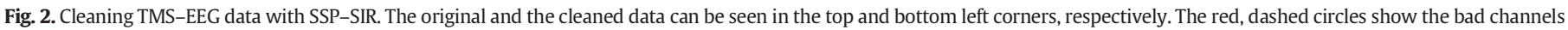
that have been removed before the SSP step. The EEG arrays are oriented so that the head of the subject is seen from above and the nose is facing to the top of the page.

sources (the same positions as in the three-layer spherical model) lying in an infinite conductive medium; the infinite-medium model lacked any boundaries that would have introduced changes in the conductivity, including the boundary between the scalp and the surrounding air. The second model was a spherical model with homogeneous conductivity, having no conductivity difference between the skull and the other compartments, but already including the confining outermost layer separating the head from the surroundings. Also in the homogeneous spherical model, the electrode and source positions were kept the same as in the three-layer model. Finally, to test the importance of EEG location accuracy, we constructed an identical model with the three-layer spherical model, except that now we changed the electrode locations, randomly distributing them onto the outermost layer. These additional models were only studied in the simulation analysis and were not used to clean the measured TMS-EEG data.

\section{Data analysis}

The TMS-EEG datasets were visually inspected for removing trials that had occasional artifacts (e.g., ocular artifacts, Ilmoniemi and Kičić, 2010). After removing the bad trials, the data were averaged to highlight the TMS-evoked responses.
Next, bad channels were visually identified (1, 8, and 16 bad channels in S1, S2, and S3, respectively) and removed from the data. The remaining channels were referenced to the average. In the later analysis, we concentrated on the time interval -50 to $200 \mathrm{~ms}$ with respect to the TMS onset.

After removing the bad trials and channels, we estimated the projection matrix $\mathbf{P}$ using Eqs. (8)-(9). For this, the TMS-EEG data were first high-pass filtered from $100 \mathrm{~Hz}$ using a fourth-order Butterworth filter and then weighted in time domain with the relative artifactamplitude envelope ${ }^{3}$ to emphasize those data points where the muscle artifact was clearly present. The SSP operator $\mathbf{P}$ was finally estimated from the singular value decomposition of the high-passed and weighted data. The artifact subspace dimensions were determined by projecting the original data onto the column vectors of $\mathbf{U}$ in Eq. (8) and observing how many of the resulting time traces showed similar time-frequency behaviour as the original data in the most artifactual channels (see Fig. 1, and for more details see the supplementary material). The

\footnotetext{
${ }^{3}$ We estimated the artifact-amplitude envelope by computing the root-mean-square value of the high-passed data in sliding 50-ms-long windows. The obtained envelope was then scaled with its maximum value, so that finally the range of the envelope was between 0 and 1.
} 
subject-specific artifact subspace was then suppressed from the TMSEEG data, see Eq. (2).

The cleaned data were reconstructed from the suppressed data using SIR, as in Eq. (4). The MNE solution, in Eq. (3), was regularized with truncated SVD by using the dimensions corresponding to the 30 largest singular values. We tested two different forward models in SIR: the realistic models and the spherical three-layer model. In the quantitative assessment of the method, we concentrated on the results obtained with the spherical head model, since often during TMS-EEG studies, individual MRIs and EEG electrode positions are not acquired.

To quantify the SSP-SIR-elicited changes in the EEG amplitude as a function of time, we computed the global mean-field amplitude (GMFA) (Lehmann and Skrandies, 1980) of the cleaned and original data. GMFA is defined as

$\operatorname{GMFA}(t)=\sqrt{\frac{1}{C} \sum_{i=1}^{C}\left[s_{i}(t)-\bar{s}(t)\right]^{2}}$,

where $s_{i}(t)$ is the signal in EEG channel $i, \bar{s}(t)=\frac{1}{C} \sum_{i=1}^{C} s_{i}(t)$, and $C$ is the number of EEG channels.

Using butterfly plots and the GMFA curve of the cleaned data, we identified four commonly observed TMS-EEG deflections from each subject: N15, P30, N45, and N100. Because the N100 response does not coincide with the muscle artifact, we used it to validate how much the genuine TMS-evoked potentials were affected by SSP-SIR. In particular, we computed the correlation coefficient (CC) between the N100 topography before and after cleaning.

Additionally, we studied whether the observed N15 topography could be produced by a cortical current source at the TMS target. By visually comparing the realistic forward models to the collected TMS navigation data, we identified the dipolar source on the anterior wall of the central sulcus that best corresponded to the location and orientation of the maximal TMS-induced electric field. We computed the CC between the topographies of the simulated dipolar source at the TMS target and the N15 response.

As mentioned above, for the main data analysis, we used the fixed SVD truncation level of 30 dimensions in the MNE solution. However, determining the correct truncation level can be cumbersome. Thus, we also wanted to study how much the recovered topographies change as a function of regularization. To quantify the sensitivity of SIR to the chosen SVD regularization, we tested all other possible regularization levels from 20 to the theoretically maximal signal dimension and then computed the topography-specific CC values of the reconstructed N15, P30, N45, and N100 responses across all the studied SVD truncation levels.

\section{Simulation analysis}

With simulations, we quantified how much SSP-SIR distorts EEG responses arising from different cortical locations. We performed the following simulation for each of our subjects. Utilizing the realistic lead field, we computed the EEG topographies produced by each of the 5124 current dipoles. The topographies were scaled so that the maximum voltage in the EEG array was $10 \mu \mathrm{V}$. We treated the simulated topographies with SSP-SIR as if they were contaminated by the same muscle artifact as in the TMS-EEG measurements. The projection matrix $\mathbf{P}$ used in SSP was obtained from the subject's real TMS-EEG dataset as explained in Theory section. In SIR, we used the three-layer spherical model and regularized MNE by including the 30 most meaningful singular directions as with the measured TMS-EEG data.

After SSP-SIR, we computed the relative error (RE) and CCs between the original simulated neuronal topographies and their post-SSP-SIR versions. We also compared the SSP-SIR-introduced distortions in the simulated topographies to the masking effects that an uncleaned muscle artifact would have; we added to each of the simulated topographies a muscle artifact topography that was obtained from the measured data by projecting the original TMS-EEG data onto the estimated artifact subspace at the time when the original signal was at its maximum GMFA.

Finally, we tested how well SIR performs with any of the three additional models, the infinite-medium model, homogeneous spherical model, and the spherical model with incorrect electrode positions, and compared the results with those obtained with the spherical threelayer model. In these comparisons, we tested all the SVD regularization levels for MNE (Eq. (3)) between 10 and the theoretical maximum in 5dimension steps, to exclude the possibility that with some of the compared models SIR would have performed well with some other regularization level than 30 dimensions.

\section{Results}

\section{Results with the measured TMS-EEG data}

All results shown in this section were obtained using the spherical three-layer model in SIR. The results were qualitatively very similar when we used the realistic head models (data not shown).

The original and cleaned TMS-EEG data of S1 are shown in Fig. 2; the corresponding data for S2 and S3 are shown in Fig. 3. Representative data measured from S1 are shown in Fig. 4 for more detail; corresponding data for S2 and S3 are shown in the supplementary material.

In all subjects, we evoked a distinctive biphasic response likely resulting from cranial muscles (see Fig. 4A); the first peak had a maximum GMFA of $16-51 \mu \mathrm{V}$ at 3.5-5.5 ms after the stimulus onset. The second deflection showed GMFA of $6-29 \mu \mathrm{V}$ peaking at $12-17 \mathrm{~ms}$. The muscle-artifact subspaces were determined to be 9-, 9-, and 6dimensional, for S1, S2, and S3, respectively. Projecting out these subspaces removed 93-99\% of all the high-pass-filtered signal.

In all subjects, SSP-SIR uncovered a deflection at 11-17 $\mathrm{ms}$ with GMFA of 1.7-2.7 $\mu \mathrm{V}$; notable deflections were observed also at 30-32, 43-50, and 85-103 ms with corresponding GMFAs of 1.3-1.8, 1.4-2.6, and $2.9-4.7 \mu \mathrm{V}$, respectively. In S1 and S2, P30 was visible only after the cleaning, and in S2, even N45 was only recognizable after SSP-SIR. The N100 response of S2 was moderately distorted by SSP-SIR (CC between the original and cleaned N100 topographies was 0.88 ), and with S1 and S3 the original and the cleaned N100 topographies matched well, resulting in CCs of 0.94 , and 0.98 .

Fig. 5 compares the N15 topography to the simulated current source at the TMS target in S1. CCs between the measured N15 topographies and the topographies of the simulated sources were $0.74,0.82$, and 0.86 . Corresponding figures for the other subjects can be found in the Supplementary material.

The results presented above and in Figs. 2-4 and in Fig. 5C were obtained by regularizing the MNE solution in Eq. (3) with SVD truncation including the 30 most significant dimensions. In all subjects, this truncation level was able to explain at least $95 \%$ of all the signal power of the suppressed data. The EEG topographies, N15, P30, N45, and N100, that were recovered with SIR were not particularly sensitive to the exact truncation level. The mean CC across all the studied regularization levels, averaged over all subjects and all topographies, was 0.94 , and over $93 \%$ of all the obtained CC values were above 0.9 .

\section{Simulation results}

The cortical maps in Fig. 6 show how SSP-SIR distorts neural EEG responses generated at different cortical sites. The maps show that especially radial sources underneath the muscle-artifact source are affected by SSP-SIR but otherwise the undesired effects stay moderate.

The post-SSP-SIR topographies matched very well the original simulated EEG topographies; in all subjects at least $84-94 \%$ of the SSP-SIRmodulated topographies had CCs $>0.90$ with respect to the original EEG topographies, with average CCs of 0.94-0.97. On the contrary, the 

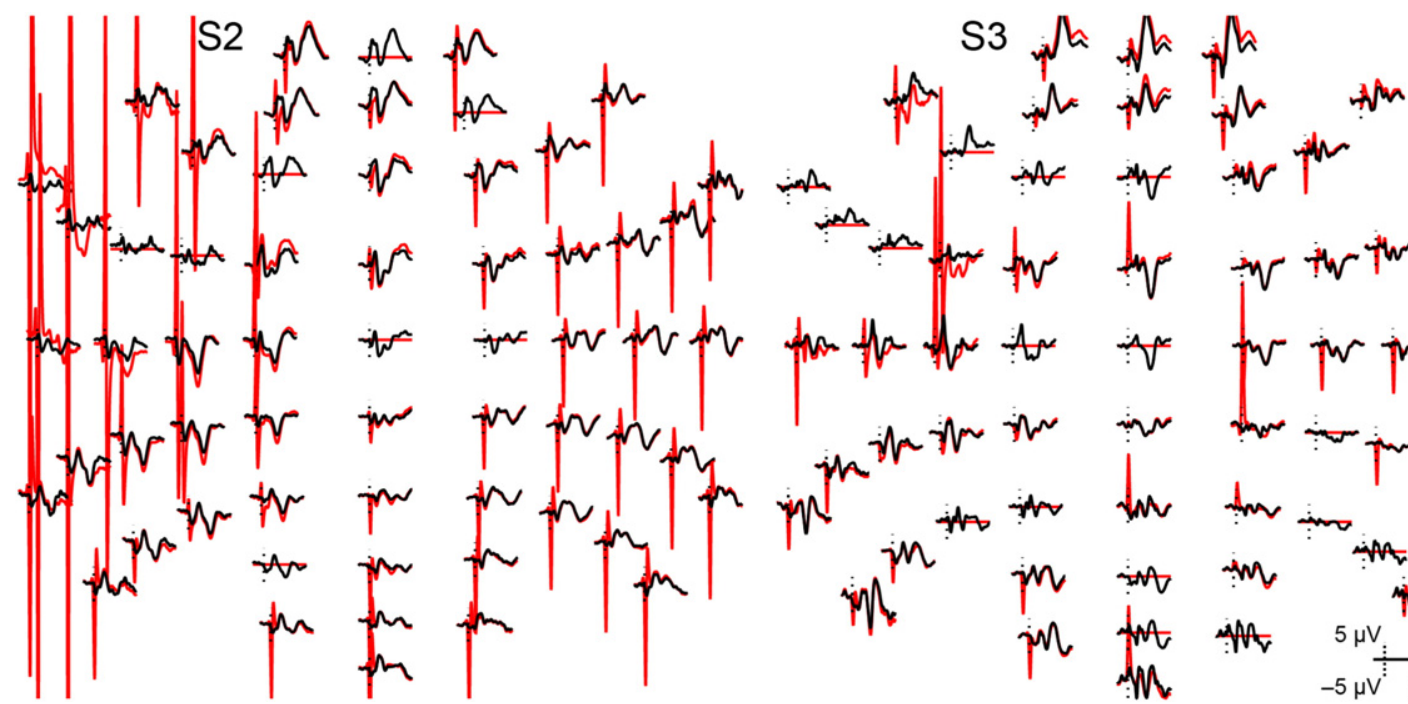

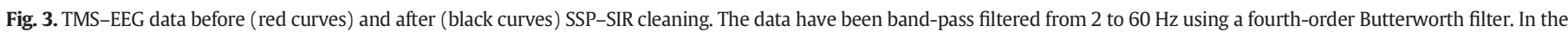
EEG arrays, the nose is facing to the top of the page.

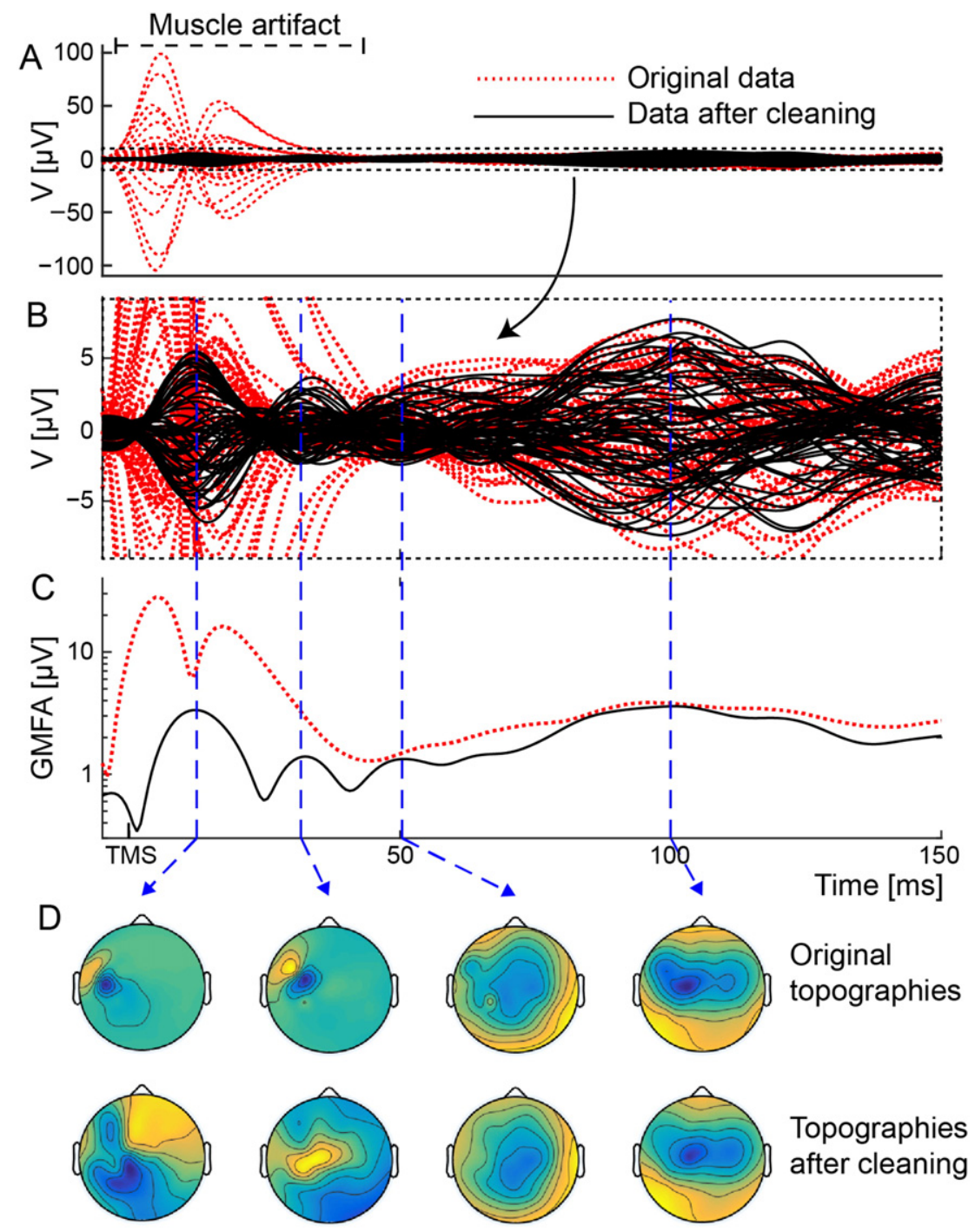

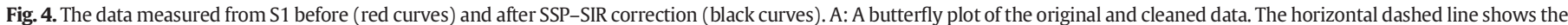

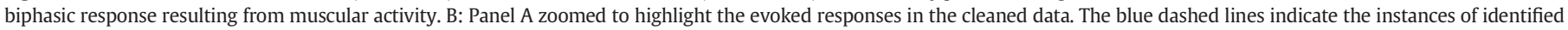

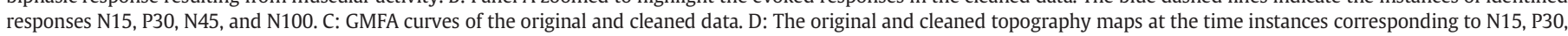
N45, and N100. 

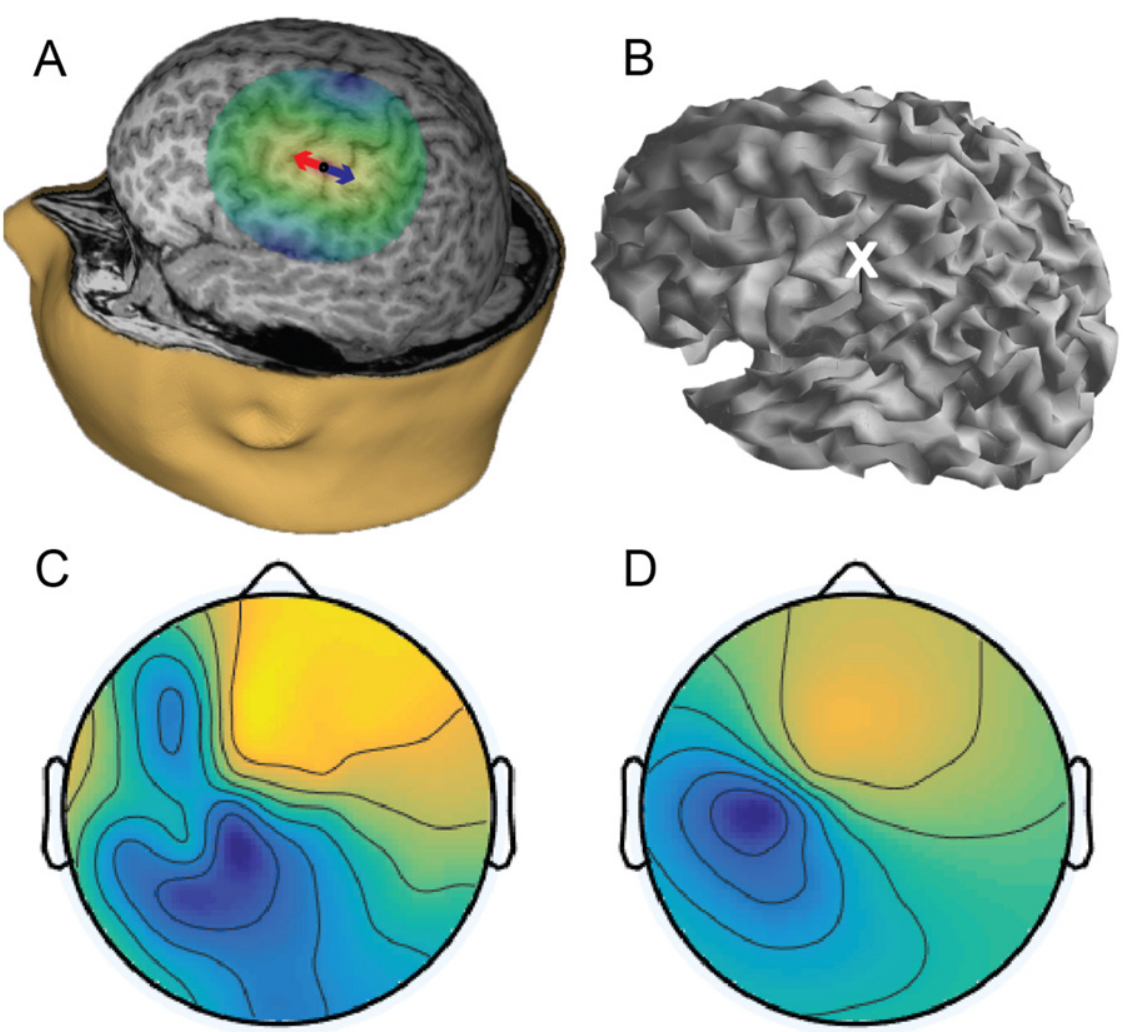

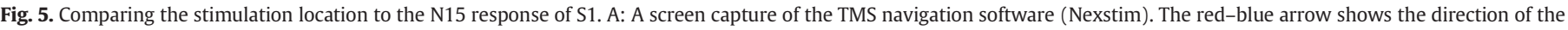

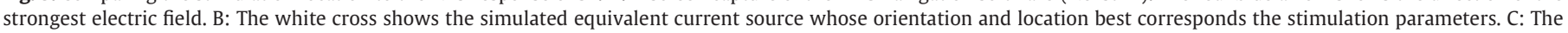
topographical pattern of the uncovered N15 response. D: The topographical pattern of the simulated source.

artifact-contaminated topographies matched very poorly the original topographies in all subjects, with an average CC of 0.17-0.23.

The average RE of the topographies after SSP-SIR was 21-36\%, depending on the subject. The added artifact caused a huge RE in the simulated topographies, varying from 700 to $1200 \%$.

When the additional models were tested in SIR, the reconstruction results clearly declined. Only the homogeneous spherical model produced to some extent tolerable results; with SVD truncation level of $40-45$ dimensions, depending on the subject, $37-82 \%$ of the SSP-SIRmodulated topographies had CC values $>0.90$. With the infinitemedium model, in all the studied cases, only $20 \%$ or less of the reconstructed topographies had CC of at least 0.90 with respect to the original EEG topographies. When the electrode positions were incorrect, only 5\% or less of the reconstructed topographies achieved the CC $>0.9$ limit.

\section{Discussion}

The muscle artifacts were clearly attenuated by SSP-SIR. As we cannot know the neuronal signals masked by the artifacts, it is impossible to judge with certainty whether the first deflections contain only brain responses or still include some residual muscle artifact. Nevertheless, the obtained results imply that we were able to uncover authentic, early TMS-evoked responses under the muscle artifacts. When stimulating M1, a similar EEG deflection at 15 ms has been measured earlier by, e.g., Komssi et al. (2004), Huber et al. (2007), and Lioumis et al. (2009). Similarly, e.g., Komssi et al. (2002), Esser et al. (2006), and Lioumis et al. (2009) have observed deflections at $30 \mathrm{~ms}$.

Especially the responses at $\sim 15 \mathrm{~ms}$ showed a broad bipolar topography that could very well be produced by a dipolar current source located under the poorly conducting skull. Additional simulations showed that a cortical current dipole located in the TMS hot spot, oriented against the precentral gyrus, produces a similar EEG pattern. Based on the orientation and low spatial frequency of the topography, it seems unlikely that the first deflections would be produced by superficial muscle tissue on the scalp.

The TMS-evoked N100 response, which was not masked by the muscle artifact, was not heavily distorted by SSP-SIR. Also, the simulations showed that the brain responses were affected only moderately by SSP-SIR. In particular, the SSP-SIR-elicited distortions were very mild compared to the effects caused by non-cleaned muscle artifacts. The brain signals that were generated on top of the lateral gyri, close to the activated muscles, were affected the most. These sources are close to the head surface and have roughly radial orientations, resulting in monopolar and relatively focal EEG field patterns. Such patterns are more likely to be correlated with the artifact subspace, and thus, affected by SSP. In Theory section, we assumed the artifact and brain subspaces to be orthogonal. The simulations show that although this assumption is not accurate for all neural sources, we can still reliably study those brain sources that have tangential orientation or that are not located directly under the cranial muscles by using SSP-SIR.

In the derivation of SSP-SIR, we made the assumption that the lowtemporal-frequency components of the muscle artifacts belong to the signal subspace defined by the high-frequency components. Although the artifacts are produced by the same muscles, we still lack knowledge about many factors, such as the orientation and the exact spatial origins of the different artifact components that might affect the subspaces. Nonetheless, the presented results suggest that the high- and lowfrequency artifact components occupy a similar signal subspace, since removal of only 6-9 dimensions that were estimated from the highpassed data was enough to suppress the visible parts of the whole artifact. 

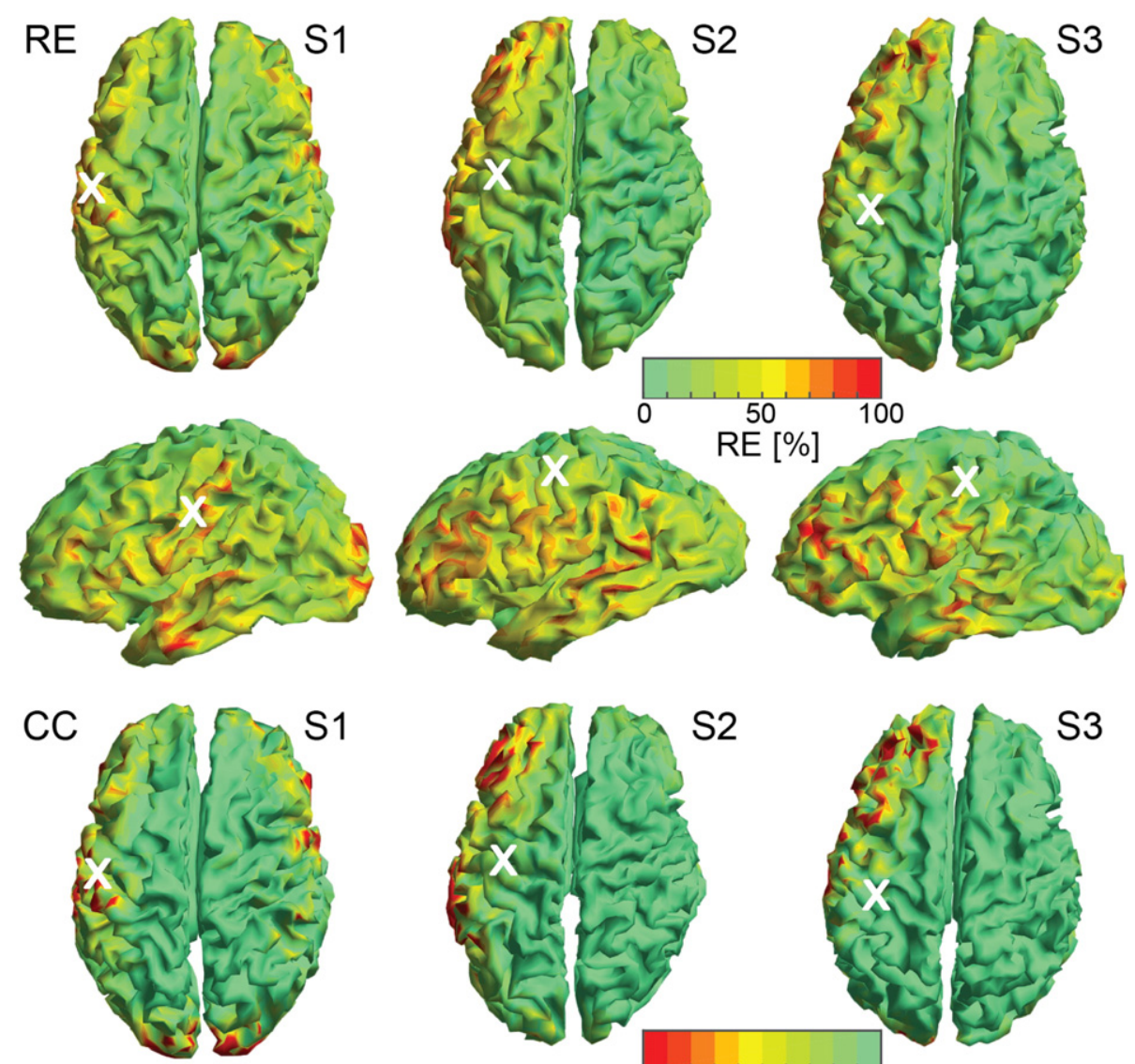

S2
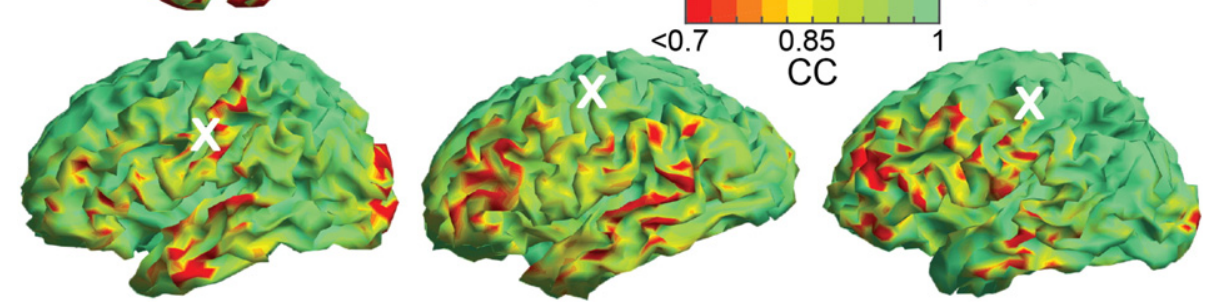

Fig. 6. The simulation results showing the SSP-SIR-caused distortion in EEG responses resulting from different cortical locations. The white crosses show the stimulation locations.

If we do not need to compute the subject-specific lead fields (e.g., for source-space analysis), it is sufficient to use SIR with a simpler conductivity model, such as the spherical three-layer model. This helps when dealing with large amounts of data and when it is not possible to build laborious realistic forward models for each subject. In the simulations, the neuronal topographies were simulated from the realistic forward models, but we were able to reconstruct the simulated topographies also using the spherical three-layer model. In addition, the results from the analysis of the real TMS-EEG data were similar, regardless of whether the realistic or spherical threelayer models were used. However, the simulations also showed that any forward model cannot be used. It seems essential to include at least the following properties in the model: confining surface that isolates the head from the surroundings, less-conductive skull that separates the scalp and the brain, and an accurate representation of the electrode array on the head.

When using SIR in this work, we regularized MNE with SVD truncation including the 30 most significant directions. This amount was able to explain at least $95 \%$ of the signal power after SSP. In future studies, one possible strategy to choose the SVD truncation level is to connect it with noise level. For example, if $\sim 10 \%$ of the data after SSP is noise, then such SVD truncation level that explains $\sim 90 \%$ of all the signal power could be chosen. Nevertheless, when we tested all the possible regularization levels from 20 upwards, ${ }^{4}$ SIR was not particularly sensitive to the exact regularization level.

It is important to note that SIR does not influence source localization. If the researchers are only interested in the inverse estimates of the TMS-EEG data, it is sufficient to use the plain SSP and take the projection into account as in Eq. (3). However, SIR recovers the neuronal EEG signals that have been disrupted by SSP so that they better resemble the original ones. This simplifies the visual and sensor-level analysis of EEG signals, enabling direct comparison between new results and those already published in the literature.

The measurements of this work were performed with the Nexstim EEG system that uses sample-and-hold circuitry to block the TMS-induced artifact (Virtanen et al., 1999) so that the pure electromagnetic artifact is not recorded. This, in addition to Nexstim's sampling specifications, might have affected the obtained results. Thus, the suggested approach should be validated when used with other EEG systems.

Here, and in the previous work by Mäki and Ilmoniemi (2011), the artifact subspace was projected out from the whole data matrix. However, this is not necessarily the best approach. Optimally, SSP-

\footnotetext{
${ }^{4} 20$ was approximately half of the theoretical dimensionality of the data after average referencing, bad-channel removal, and SSP.
} 
SIR should be used to recover brain responses only at those times when the muscle artifacts are present but otherwise the data can be kept untouched.

\section{Conclusion}

We have developed SSP-SIR method, which is able to considerably reduce muscle artifacts in TMS-evoked EEG while mostly preserving the underlying neuronal EEG. The methodology was demonstrated to work with simulations and with real data. SSP-SIR helps in studies of artifactual TMS-EEG data, extending the range of cortical areas that can be directly studied.

\section{Conflict-of-interest statement}

Risto J. Ilmoniemi is the founder, advisor, and a minority shareholder of Nexstim Plc.

\section{Acknowledgments}

This study was supported by the Academy of Finland (283105) and the Finnish Cultural Foundation (00150064 and 00161149). We want to thank Javier Gómez Bolaños who helped us to calibrate the white-noise level appropriately using a phantom ear. We also thank Niko Mäkelä, who assisted in segmentation, and Elina Mäkelä, who helped in parts of the measurements.

\section{Appendix A}

We consider a spherical model for the head consisting of three concentric layers: brain, skull, and scalp, whose outer radii and conductivities are denoted by $\left\{r_{1}, \sigma_{1}\right\},\left\{r_{2}, \sigma_{2}\right\}$, and $\left\{r_{3}, \sigma_{3}\right\}$, respectively. To calculate the lead field in this geometry, we must find the potential $V\left(\mathbf{r}, \mathbf{r}_{\mathrm{Q}}, \mathbf{Q}\right)$ at a scalp location $\mathbf{r}$ produced by a current dipole with a moment $\mathbf{Q}$ lying at location $\mathbf{r}_{\mathrm{Q}}\left(\left|\mathbf{r}_{\mathrm{Q}}\right| \leq r_{1}\right)$.

The expression for $V$, see, e.g., Zhang (1995), can be written for the three-layer case as

$$
\begin{aligned}
& V\left(\mathbf{r}, \mathbf{r}_{\mathrm{Q}}, \mathbf{Q}\right) \\
& \quad=\frac{1}{4 \pi \sigma_{3}} \sum_{n=1}^{\infty} \gamma_{n}\left[\mathbf{Q} \cdot \hat{\mathbf{r}}_{\mathrm{Q}} n P_{n}\left(\hat{\mathbf{r}} \cdot \hat{\mathbf{r}}_{\mathrm{Q}}\right)+\left(\mathbf{Q} \times \hat{\mathbf{r}}_{\mathrm{Q}}\right) \cdot\left(\hat{\mathbf{r}} \times \hat{\mathbf{r}}_{\mathrm{Q}}\right) P_{n}^{\prime}\left(\hat{\mathbf{r}} \cdot \hat{\mathbf{r}}_{\mathrm{Q}}\right)\right],
\end{aligned}
$$

where $\hat{\mathbf{r}}=\mathbf{r} / r, r=|\mathbf{r}|, \hat{\mathbf{r}}_{\mathrm{Q}}=\mathbf{r}_{\mathrm{Q}} / r_{\mathrm{Q}}, r_{\mathrm{Q}}=\left|\mathbf{r}_{\mathrm{Q}}\right|$, and $P_{n}$ is the order- $n$ Legendre polynomial and $P_{n}^{\prime}$ its derivative. The coefficients $\gamma_{n}$ are given by

$\gamma_{n}=\frac{r_{3}^{n} r_{\mathrm{Q}}^{n-1}}{c_{1} r_{3}^{2 n+1}-c_{2} r_{2}^{2 n+1}}$,

where

$$
\begin{aligned}
c_{1}= & \frac{n(n+1)}{(2 n+1)^{3}}\left[\left(1+\frac{n}{n+1} \frac{\sigma_{2}}{\sigma_{3}}\right)\left(n+1+n \frac{\sigma_{1}}{\sigma_{2}}\right)\right. \\
& \left.+n\left(1-\frac{\sigma_{2}}{\sigma_{3}}\right)\left(1-\frac{\sigma_{1}}{\sigma_{2}}\right)\left(\frac{r_{1}}{r_{2}}\right)^{2 n+1}\right]
\end{aligned}
$$

and

$$
\begin{aligned}
c_{2}= & \frac{n(n+1)}{(2 n+1)^{3}}\left[\left(1-\frac{\sigma_{2}}{\sigma_{3}}\right)\left(n+1+n \frac{\sigma_{1}}{\sigma_{2}}\right)\right. \\
& \left.+\left(n+(n+1) \frac{\sigma_{2}}{\sigma_{3}}\right)\left(1-\frac{\sigma_{1}}{\sigma_{2}}\right)\left(\frac{r_{1}}{r_{2}}\right)^{2 n+1}\right] .
\end{aligned}
$$

\section{Appendix B. Supplementary data}

Supplementary data to this article can be found online at http://dx. doi.org/10.1016/j.neuroimage.2016.05.028.

\section{References}

Baillet, S., Mosher, J.C., Leahy, R.M., 2001. Electromagnetic brain mapping. IEEE Signal Process. Mag. 18, 14-30.

Barker, A.T., Jalinous, R., Freeston, I.L., 1985. Non-invasive magnetic stimulation of human motor cortex. Lancet 325, 1106-1107.

Burghoff, M., Nenonen, J., Trahms, L., Katila, T., 2000. Conversion of magnetocardiographic recordings between two different multichannel SQUID devices. IEEE Trans. Biomed. Eng. 47, 869-875.

Buzsáki, G., Draguhn, A., 2004. Neuronal oscillations in cortical networks. Science 304, 1926-1929.

Cohen, D. Cuffin, B.N., 1983. Demonstration of useful differences between magnetoencephalogram and electroencephalogram. Electroencephalogr. Clin. Neurophysiol. 56, 38-51.

Cona, F., Zavaglia, M., Massimini, M., Rosanova, M., Ursino, M., 2011. A neural mass model of interconnected regions simulates rhythm propagation observed via TMS-EEG. NeuroImage 57, 1045-1058.

Delorme, A., Makeig, S., 2004. EEGLAB: an open source toolbox for analysis of single-trial EEG dynamics including independent component analysis. J. Neurosci. Methods 134, 9-21.

Esser, S.K., Huber, R., Massimini, M., Peterson, M.J., Ferrarelli, F., Tononi, G., 2006. A direct demonstration of cortical LTP in humans: a combined TMS/EEG study. Brain Res. Bull. 69, 86-94.

Fang, Q., Boas, D.A., 2009. Tetrahedral mesh generation from volumetric binary and grayscale images. IEEE International Symposium on Biomedical Imaging: From Nano to Macro, 2009, pp. 1142-1145 (ISBI'09).

Farzan, F., Barr, M.S., Hoppenbrouwers, S.S., Fitzgerald, P.B., Chen, R., Pascual-Leone, A., Daskalakis, Z.J., 2013. The EEG correlates of the TMS-induced EMG silent period in humans. NeuroImage 83, 120-134.

Fischl, B., 2012. FreeSurfer. Neurolmage 62, 774-781.

Gonçalves, S.I., de Munck, J.C., Verbunt, J.P.A., Bijma, F., Heethaar, R.M., Lopes da Silva, F., 2003. In vivo measurement of the brain and skull resistivities using an EIT-based method and realistic models for the head. IEEE Trans. Biomed. Eng. 50, 754-767.

Gramfort, A., Luessi, M., Larson, E., Engemann, D.A., Strohmeier, D., Brodbeck, C., Parkkonen, L., Hämäläinen, M.S., 2014. MNE software for processing MEG and EEG data. Neurolmage 86, 446-460.

Hämäläinen, M.S., Ilmoniemi, R.J., 1994. Interpreting magnetic fields of the brain: minimum norm estimates. Med. Biol. Eng. Comput. 32, 35-42.

Hernandez-Pavon, J.C., Metsomaa, J., Mutanen, T., Stenroos, M., Mäki, H., Ilmoniemi, R.J., Sarvas, J., 2012. Uncovering neural independent components from highly artifactual TMS-evoked EEG data. J. Neurosci. Methods 209, 144-157.

Huber, R., Esser, S.K., Ferrarelli, F., Massimini, M., Peterson, M.J., Tononi, G., 2007. TMSinduced cortical potentiation during wakefulness locally increases slow wave activity during sleep. PLoS One 2, e276.

Ilmoniemi, R.J., Kičić, D., 2010. Methodology for combined TMS and EEG. Brain Topogr. 22, 233-248.

Ilmoniemi, R.J., Virtanen, J., Ruohonen, J., Karhu, J., Aronen, H.J., Näätänen, R., Katila, T., 1997. Neuronal responses to magnetic stimulation reveal cortical reactivity and connectivity. Neuroreport 8, 3537-3540.

Julkunen, P., Säisänen, L., Danner, N., Niskanen, E., Hukkanen, T., Mervaala, E., Könönen, M., 2009. Comparison of navigated and non-navigated transcranial magnetic stimulation for motor cortex mapping, motor threshold and motor evoked potentials. Neurolmage 44, 790-795.

Komssi, S. Aronen, H.J., Huttunen, J., Kesäniemi, M., Soinne, L., Nikouline, V.V., Ollikainen, M., Roine, R.O., Karhu, J., Savolainen, S., Ilmoniemi, R.J., 2002. Ipsi- and contralateral EEG reactions to transcranial magnetic stimulation. Clin. Neurophysiol. 113, 175-184.

Komssi, S., Kähkönen, S., Ilmoniemi, R.J., 2004. The effect of stimulus intensity on brain responses evoked by transcranial magnetic stimulation. Hum. Brain Mapp. 21, 154-164.

Korhonen, R.J., Hernandez-Pavon, J.C., Metsomaa, J., Mäki, H., Ilmoniemi, R.J., Sarvas, J., 2011. Removal of large muscle artifacts from transcranial magnetic stimulationevoked EEG by independent component analysis. Med. Biol. Eng. Comput. 49, 397-407.

Lai, Y., van Drongelen, W., Ding, L., Hecox, K., Towle, V., Frim, D.M., He, B., 2005. Estimation of in vivo human brain-to-skull conductivity ratio from simultaneous extra-and intra-cranial electrical potential recordings. Clin. Neurophysiol. 116, 456-465.

Lehmann, D., Skrandies, W., 1980. Reference-free identification of components of checkerboard-evoked multichannel potential fields. Electroencephalogr. Clin. Neurophysiol. 48, 609-621.

Lioumis, P., Kičić, D., Savolainen, P., Mäkelä, J.P., Kähkönen, S., 2009. Reproducibility of TMS-evoked EEG responses. Hum. Brain Mapp. 30, 1387-1396.

Mäki, H., Ilmoniemi, R.J., 2011. Projecting out muscle artifacts from TMS-evoked EEG. Neurolmage 54, 2706-2710.

Massimini, M., Ferrarelli, F., Huber, R., Esser, S.K., Singh, H., Tononi, G., 2005. Breakdown of cortical effective connectivity during sleep. Science 309, 2228-2232.

Mutanen, T., Mäki, H., Ilmoniemi, R.J., 2013. The effect of stimulus parameters on TMSEEG muscle artifacts. Brain Stimul. 6, 371-376.

Nikouline, V., Ruohonen, J., Ilmoniemi, R.J., 1999. The role of the coil click in TMS assessed with simultaneous EEG. Clin. Neurophysiol. 110, 1325-1328. 
Nikulin, V.V., Kičić, D., Kähkönen, S., Ilmoniemi, R.J., 2003. Modulation of electroencephalographic responses to transcranial magnetic stimulation: evidence for changes in cortical excitability related to movement. Eur. J. Neurosci. 18, 1206-1212.

Numminen, J., Ahlfors, S., Ilmoniemi, R., Montonen, J., Nenonen, J., 1995. Transformation of multichannel magnetocardiographic signals to standard grid form. IEEE Trans. Biomed. Eng. 42, 72-78.

Nunez, P.L., Silberstein, R.B., 2000. On the relationship of synaptic activity to macroscopic measurements: does co-registration of EEG with fMRI make sense? Brain Topogr. 13, 79-96.

Rogasch, N.C., Thomson, R.H., Daskalakis, Z.J., Fitzgerald, P.B., 2013. Short-latency artifacts associated with concurrent TMS-EEG. Brain Stimul. 6, 868-876.

Rogasch, N.C., Thomson, R.H., Farzan, F., Fitzgibbon, B.M., Bailey, N.W., Hernandez-Pavon, J.C., Daskalakis, Z.J., Fitzgerald, P.B., 2014. Removing artefacts from TMS-EEG recordings using independent component analysis: importance for assessing prefrontal and motor cortex network properties. Neurolmage 101, 425-439.

Rosanova, M., Casali, A., Bellina, V., Resta, F., Mariotti, M., Massimini, M., 2009. Natural frequencies of human corticothalamic circuits. J. Neurosci. 29, 7679-7685.

Rossini, P.M., Barker, A.T., Berardelli, A., Caramia, M.D., Caruso, G., Cracco, R.Q. Dimitrijević, M., Hallett, M., Katayama, Y., Lücking, C.H., et al., 1994. Non-invasive electrical and magnetic stimulation of the brain, spinal cord and roots: basic principles and procedures for routine clinical application. Report of an IFCN committee. Electroencephalogr. Clin. Neurophysiol. 91, 79-92.

Rush, S., Driscoll, D.A., 1969. EEG electrode sensitivity-an application of reciprocity. IEEE Trans. Biomed. Eng. 16, 15-22.
Shattuck, D.W., Leahy, R.M., 2002. Brainsuite: an automated cortical surface identification tool. Med. Image Anal. 6, 129-142.

Stenroos, M., Nummenmaa, A., 2016. Incorporating and compensating cerebrospinal fluid in surface-based forward models of magneto- and electroencephalography. bioRxiv 037788.

Stenroos, M., Sarvas, J., 2012. Bioelectromagnetic forward problem: isolated source approach revis(it)ed. Phys. Med. Biol. 57, 3517-3536.

ter Braack, E., de Jonge, B., van Putten, M.J., 2013. Reduction of TMS induced artifacts in EEG using principal component analysis. IEEE Trans. Neural Syst. Rehabil. Eng. 21, 376-382.

ter Braack, E.M., de Vos, C.C., van Putten, M.J., 2015. Masking the auditory evoked potential in TMS-EEG: a comparison of various methods. Brain Topogr. 28, 520-528.

Uusitalo, M.A., Ilmoniemi, R.J., 1997. Signal-space projection method for separating MEG or EEG into components. Med. Biol. Eng. Comput. 35, 135-140.

Uutela, K., Taulu, S., Hämäläinen, M., 2001. Detecting and correcting for head movements in neuromagnetic measurements. Neurolmage 14, 1424-1431.

Virtanen, J., Ruohonen, J., Näätänen, R., Ilmoniemi, R.J., 1999. Instrumentation for the measurement of electric brain responses to transcranial magnetic stimulation. Med. Biol. Eng. Comput. 37, 322-326.

Zhang, Z., 1995. A fast method to compute surface potentials generated by dipoles within multilayer anisotropic spheres. Phys. Med. Biol. 40, 335-349. 